\title{
Effectiveness of Bibliotherapy on Quality of Life, Distress and Depression among Chronic Leg and Foot Ulcer
}

\author{
Kirupa. $\mathbf{P}^{1}$, Shrinivasa Bhat ${ }^{2}$ \\ ${ }^{1}$ Principal, Indra Ganesan College of Nursing, (TNMGRMU), Trrichy-620012 \\ ${ }^{2}$ Professor, Department of Psychiatry, K. S. Hegde Medical Academy, Nitte Deemed to be University, \\ Deralakatte, Mangalore-18. \\ Corresponding Author: Kirupa. P
}

\begin{abstract}
Chronic leg and foot ulcer is an endangering health issue secondary to chronic venous insufficiency and Diabetes Mellitus. Apart from physical problems, psychological and emotional disturbances were arising as a co morbid conditions, which interfere with recovery and it could be managed by bibliotherapy. Study was focused to ascertain the effectiveness of bibliotherapy on quality of life, psychological distress and depression of these patients in an Indian context. Randomized controlled trial comparison with parallel group design was chosen. Interventional group received usual care along with bibliotherapy and control group received only usual care. Data were collected pretest, post test $\left(1^{\text {st }}\right.$ week), I follow up ( $2^{\text {nd }}$ week $)$ and II follow up ( $3^{\text {rd }}$ week). Cardiff wound impact schedule, Kessler's psychological distress scale and Beck depression inventory were used. Bibliotherapy was prepared by the researcher based on literatures and qualitative study. Setting was limited to the selected hospitals in Mangalore, India. Power analysis estimated sample size as 50 in interventional group and 50 in control group. Findings imply that compared with controls, patients who received bibliotherapy demonstrated improved quality of life, reduced psychological distress and depression. Conclusively, it recommends psychosocial perspectives of these clients should not be neglected and supporting the therapeutic benefit of bibliotherapy in chronic physical illnesses to reduce mental health burden.
\end{abstract}

Key Words: Quality of life, Psychological Distress, Depression, Bibliotherapy, Leg and Foot ulcer.

\section{INTRODUCTION}

Chronic Leg and foot ulcers are often represented as long-term health care issues. Immobility, sleeplessness, social isolation, pain, and fear of disability negatively affect the mental health and quality of life (QoL). Psychosocial impacts on patients with chronic leg wounds are from impaired health related quality of life, stress, distress, unrevealed depression, symptoms of depression and finally evolving into major depression. It is very essential to focus on the psychological perspective of these patients. The Indian healthcare system emphasizes to target their mental health issues ${ }^{(1) .}$ Bibliotherapy is also called as 'self-help books'. The American Library Association defined bibliotherapy as selected reading materials used as an adjuvant therapy in the context of medicine and psychiatry by guiding solutions to personal problems ${ }^{(2)}$

\section{Need for the study}

A study on 318 patients with venous leg ulcers showed that poor quality of life was manifested in physical components (mean-33.16) and mental health components (mean- 46.61). Most frequent symptoms were sleep disturbances $(80 \%)$, 
pain $(74 \%)$; and lower limb swelling $(67 \%)^{(3)}$. Bibliotherapy is also used as an adjuvant therapy for mild to moderate degree of depression and psychological distress. Bibliotherapy is an inexpensive method. In Switzerland, a study on internetbased self- help protocol was carried out for seventy-six individuals. The findings suggested that and bibliotherapy was beneficial to reduce moderate depressive symptoms ${ }^{(4)}$. The researcher prepared a book based on the needs of patients suffering from chronic leg and foot ulcer to evaluate the effectiveness on quality of life, Distress and depression.

\section{Objectives}

- To assess the pre-test level of quality of life, psychological distress, and depression in both the interventional group and the control group.

- To evaluate the effectiveness of bibliotherapy.

\section{Hypothesis}

$\mathrm{H} 1$ - There will be significant difference in the post- test level of quality of life between the interventional group and the control group at 0.05 level of significance.

$\mathrm{H} 2$ - There will be significant difference in the post- test level of psychological distress between the interventional group and the control group at 0.05 level of significance.

H3 - There will be significant difference in the post- test level of Depression between the interventional group and the control group at 0.05 level of significance.

\section{LITERATURE SURVEY}

The literature survey was presented and organized on the basis of impact of chronic lower extremity wounds and influence of bibliotherapy. A systematic review conducted by the researcher analyzed studies related to the impact of chronic leg and foot ulcer during 2010-2014 (5).

\section{Literature related to Impact of chronic lower extremity wounds \\ An observational study was} conducted on diabetic foot ulcer for a period of 2 years among 293 patients in India. The result shows that $7.5 \%$ of the subjects reported as hopeless to live, $22.06 \%$ had depression, and $7.58 \%$ had anxiety. Diabetic foot ulceration has an impact on occupation. About $13.7 \%$ were retired, $16.55 \%$ were unable to meet their economic needs, and $12.06 \%$ had lost their job due to immobility and repeated hospitalization. After amputation, $1.03 \%$ had post-traumatic stress disorder and $5.51 \%$ had post- operative delirium ${ }^{(6)}$.

\section{Literature related to the impact chronic leg and foot ulcer}

In Switzerland, a study on internetbased self- help protocol was carried out for ten-week duration. Seventy-six individuals were grouped in to guided self- help group, unguided self- help group, and control group. A structured diagnostic telephonic interview was done before the selection of the subjects. The post- test was attended by $91 \%$, and after 6 months, $78 \%$ attended the follow-up. The study concluded that internet-based self- help on quality of life and depression could be effective with or without guidance $^{(7)}$.

\section{MATERIAL AND METHODS}

As per the study framework, randomized control trial comparison with parallel group was adopted. The trial included the control group, administration of the intervention, and randomization. The interventional group received standard care along with bibliotherapy, while the control group received only standard care. Standard care includes drugs and wound care. The pre-test was done on the first day. Post- test was administered in the first week, I followup in the second week, and II follow- up in the third week for both the interventional and control group. The present study was conducted in the surgical ward of selected hospitals in Mangalore, Karnataka. 
Sample: The patients who fulfill the inclusion and exclusion criteria

Inclusion Criteria: This included patients who

- Had been diagnosed with either diabetic foot ulcer or venous leg ulcer or both.

- Were between 30 -65years of age.

- Had suffered recurrent diabetic foot ulcer or venous leg ulcer.

- Were able to understand and speak either Kannada, Malayalam or English

- Had the site of ulcer below knee.

- Had undergone any one of the surgical treatments of incision and drainage, debridement, disarticulation or Trendelenburg procedure.

- Had completed secondary school education. $\left(10^{\text {th }}\right.$ Standard $)$

Exclusion criteria: This excluded patients who diagnosed with chronic alcoholism and alcohol dependent syndrome.

\section{Instruments}

Instruments of the study are socio demographic variables, Wound profile, Cardiff wound impact schedule, Kessler's psychological distress scale And Beck depression inventory. The Crohnbach alpha coefficient implied that the translated version of the instruments in both the languages was significantly reliable. Permission was obtained from the concerned author to use the CWIS. Its maximum score is 320. Kessler's Psychological Distress Scale (K10) is one of the special scales constructed by Kessler. Permission was obtained from the author to modify the assessment duration from 30 days to 7 days. Validation related to the change of days was done after consultation with experts in the field of clinical psychology. Its maximum score is 50. Beck Depression Inventory-II (BDI-II) measures the severity of depressive symptoms subjectively. Depressive symptoms composed in this instrument are based on the thoughts or cognitions of the individual. Its maximum score is 63 with clinical borderline as 20 .

\section{Intervention}

The Intervention bibliotherapy book was prepared based on the qualitative study and supportive literature. Content validation of the book was done by experts in the fields of Psychology, Psychiatry, Surgery, and Nursing. The book was translated into Malayalam and Kannada and validated by literature experts. The pre- testing of the book was done by offering it to a sample of patients to check for clarity. The Bibliotherapy (self-help book) was prepared to the level which is understandable by an eighth standard student.

\section{Description about the Intervention}

The intervention administered to the patients was need- based bibliotherapy. It is a contemporary approach to help patients deal with their problems, both mentally and physically. The book was administered in the paper form. Concepts of the book are Creating knowledge, Motivating self- care, Caution about complications, balancing negative emotions, Removing negative thoughts and Management of day- to- day battle with chronic leg wounds. A pilot study reported that the intervention was significantly effective on the quality of life, psychological distress, and depression of the patients with chronic leg and foot ulcer ${ }^{(8)}$.

\section{Data Collection Procedure}

Informed consent was obtained from each participant. The pre-test was conducted on the day of selection. Bibliotherapy along with standard care was administrated individually to patients, who belonged to the interventional group. The control group patients were received only standard care. The duration of the intervention was one week .Post- test was administrated after the one week period. The same instruments were used to assess the effectiveness of the intervention during the follow- up in the second (I follow- up) and third (II followup) week. The same procedure was followed for the control group patients. The complete data was collected in the hospital setting. Programme participation was 
monitored in the interventional group. All patients received the booklet at the time of their discharge.

\section{RESULTS}

Percentage, mean, and standard deviation were presented about the sociodemographic characteristics and baseline level of QoL, psychological distress, and depression. The findings are presented in graphs and tables. An outcome point of view, bibliotherapy on the quality of life, depression, and psychological distress was analyzed. The results are represented by using Microsoft Excel 2007 and SPSS 16 version. Chi- square analysis, independent ' $t$ ' test, repeated measures ANOVA (analysis of variance), Bonferroni correction and Pearson product moment correlation was applied ${ }^{(11),(10)}$.

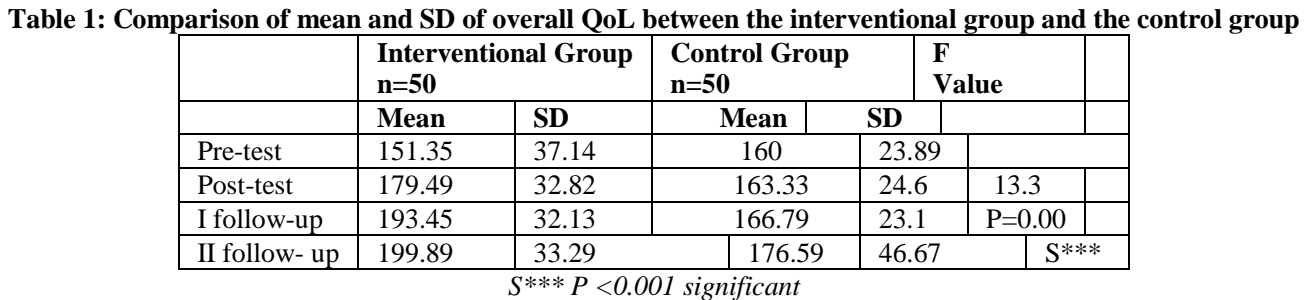

In table (1), The $\mathrm{F}_{(3,147)}$ value obtained was 13.3 at 0.05 level of significance for interaction effect of various data collection points vs. two groups. The research hypothesis, H1 was accepted at 0.05 level of significance. It shows that there is significant increase in the level of overall quality of life after the administration of bibliotherapy on the interventional group. The multiple points of comparison (Bonferroni correction) represent that clinically there is a remarkable mean difference in the overall QoL between the interventional and control groups (pretest with posttest 15.68, I follow up 24.39 II follow up 32.51 and also posttest with I follow up 8.71 and II follow up 16.83)

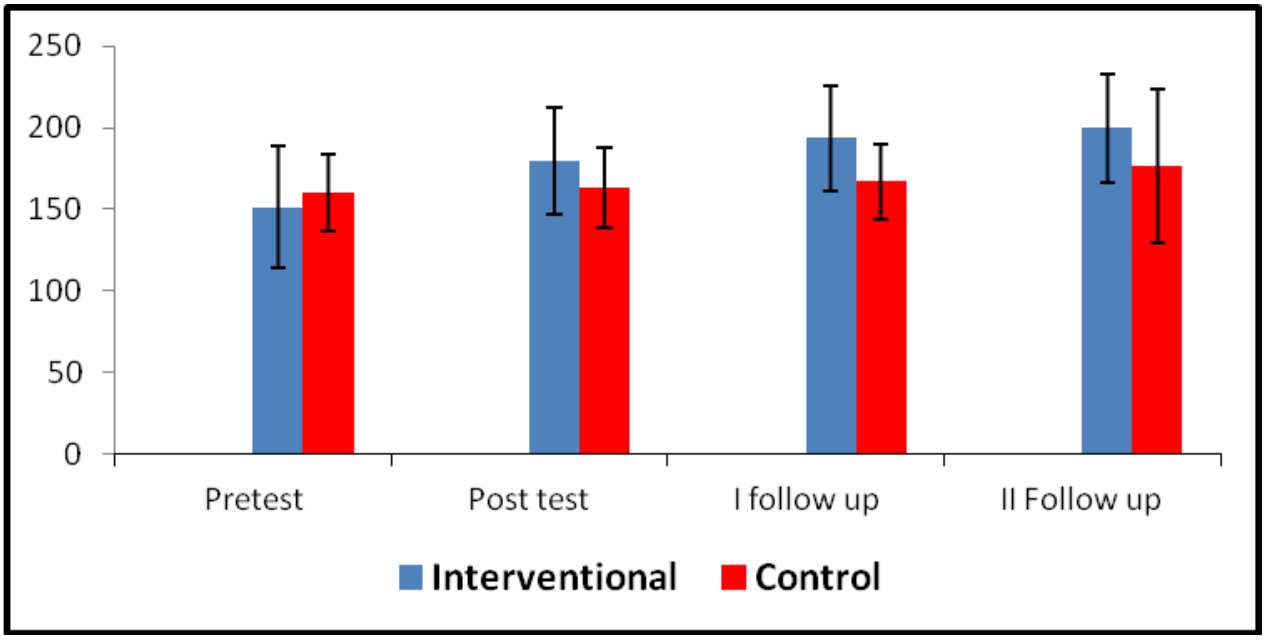

Figure-1 Error plot diagram on overall QOL at various points vs. groups.

Table 2: Comparison of mean and SD of psychological distress between the interventional and control groups.

\begin{tabular}{|c|c|c|c|c|c|}
\hline & \multicolumn{2}{|c|}{$\begin{array}{c}\text { Interventional Group } \\
\mathbf{n = 5 0}\end{array}$} & $\begin{array}{l}\text { Control Group } \\
\mathbf{n}=50\end{array}$ & & \multirow[t]{2}{*}{$\begin{array}{l}\text { F } \\
\text { Value }\end{array}$} \\
\hline & \begin{tabular}{l|l} 
Mean \\
\end{tabular} & SD & \begin{tabular}{l|l|} 
& Mean \\
\end{tabular} & SD & \\
\hline Pre-test & 29.3 & 6.19 & 30.94 & 3.62 & \\
\hline Post- test & 22.68 & 5.78 & 31.62 & 3.89 & 92.04 \\
\hline I follow-up & 19.59 & 4.99 & 33.4 & 3.21 & $\mathrm{P}=0.00$ \\
\hline II follow- up & 18.17 & 5.5 & 33.61 & 3.4 & $\mathrm{~S}^{* * *}$ \\
\hline
\end{tabular}



foot ulcer.

In Table (2), repeated measures ANOVA was done to compare the mean of psychological distress. The $\mathrm{F}_{(3,147)}$ value obtained was 92.04 at 0.05 level of significance for interaction effect of various data collection points vs. groups. The research hypothesis, $\mathrm{H} 2$ was accepted at 0.05 level of significance. It shows that there is a gradual decrease in the level of distress after the administration of bibliotherapy on the interventional group. In the control group, psychological distress gradually increased in the post-test, I follow- up, and II follow-up, than in the interventional group. Bibliotherapy is significantly effective in reducing psychological distress. The partial eta square value 0.6 shows that magnitude of mean difference was above medium (60\%). The multiple comparisons (Bonferroni correction) of mean difference within the different points of the data collection with the Bonferroni correction. The multiple points of comparison represent that clinically there is a remarkable mean difference of psychological distress between the interventional and control groups (pretest with posttest -2.97, I follow up-3.44 and II follow up -4.06 ).

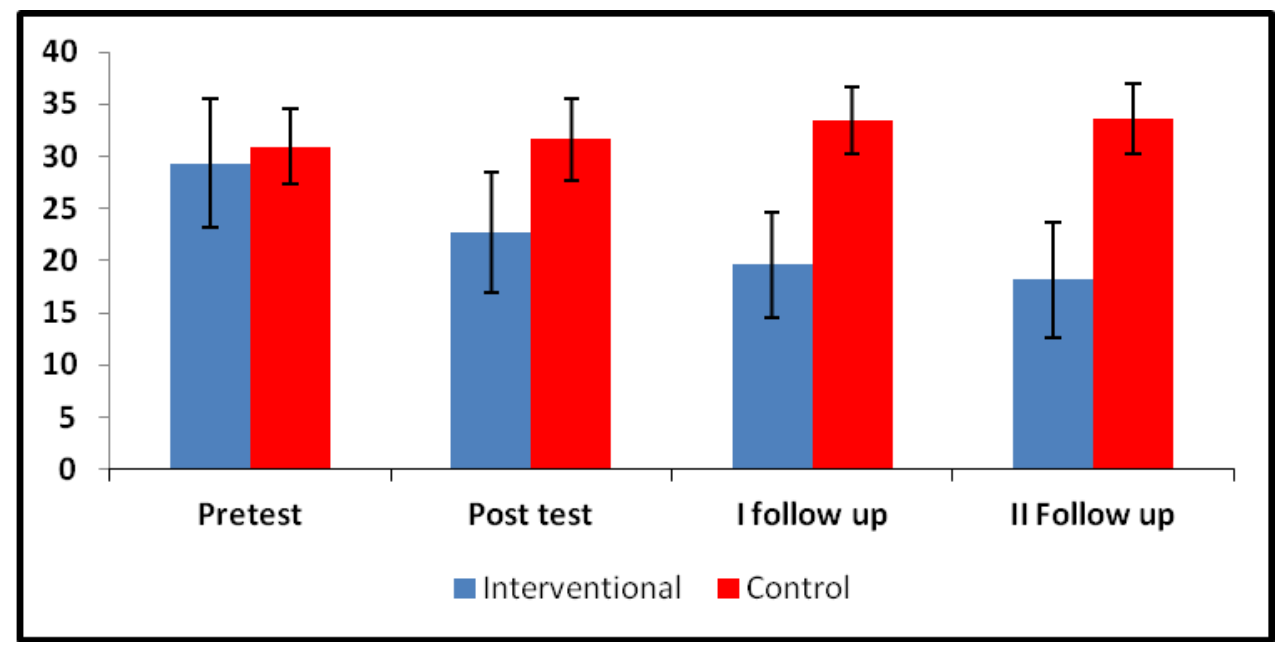

Figure- 2 : Error plot diagram on distress at various points vs. groups.

Table 3: Comparison of mean and SD of depression between the interventional and control groups

\begin{tabular}{|c|c|c|c|c|c|}
\hline & \multicolumn{2}{|c|}{$\begin{array}{l}\text { Interventional Group } \\
\quad \mathbf{n}=\mathbf{5 0}\end{array}$} & $\begin{array}{c}\text { Control Group } \\
\mathbf{n}=\mathbf{5 0}\end{array}$ & 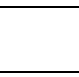 & \multirow[t]{2}{*}{$\begin{array}{l}\mathbf{F} \\
\text { Value }\end{array}$} \\
\hline & \begin{tabular}{l|l} 
& Mean
\end{tabular} & SD & \begin{tabular}{l|l} 
& Mean
\end{tabular} & SD & \\
\hline Pre-test & 22.88 & 5.03 & 21.38 & 4.04 & \\
\hline Post -test & 19 & 5.45 & 20.72 & 3.69 & 32.3 \\
\hline I follow- up & 16.59 & 3.81 & 21.18 & 3.52 & $\mathrm{P}=0.00$ \\
\hline II follow- up & 16.43 & 4.06 & 21.21 & 4.71 & $\mathrm{~S}^{* * * *}$ \\
\hline
\end{tabular}

In Table (3), repeated measures ANOVA was done to compare the mean of depression at 0.05 level of significance. The research hypothesis, H3 was accepted at 0.05 level of significance. It shows that there is a significant decrease in the level of depression after the administration of bibliotherapy on the interventional group. The partial eta square value 0.3 depicted that the magnitude of mean difference was just above medium (30\%). In the control group, the depression was the same in the post-test and I and II follow-up, than in the interventional group. Bibliotherapy is significantly effective in reducing depression in patients suffering from chronic leg and foot ulcer.

The multiple comparisons (Bonferroni Correction) of mean difference within different points of the data collection with the Bonferroni correction. The multiple points of comparison represent that clinically there is remarkable mean difference in depression between the 
interventional and control groups (pretest follow up 3.31). with posttest 2.27 , I follow up 3.24 and II

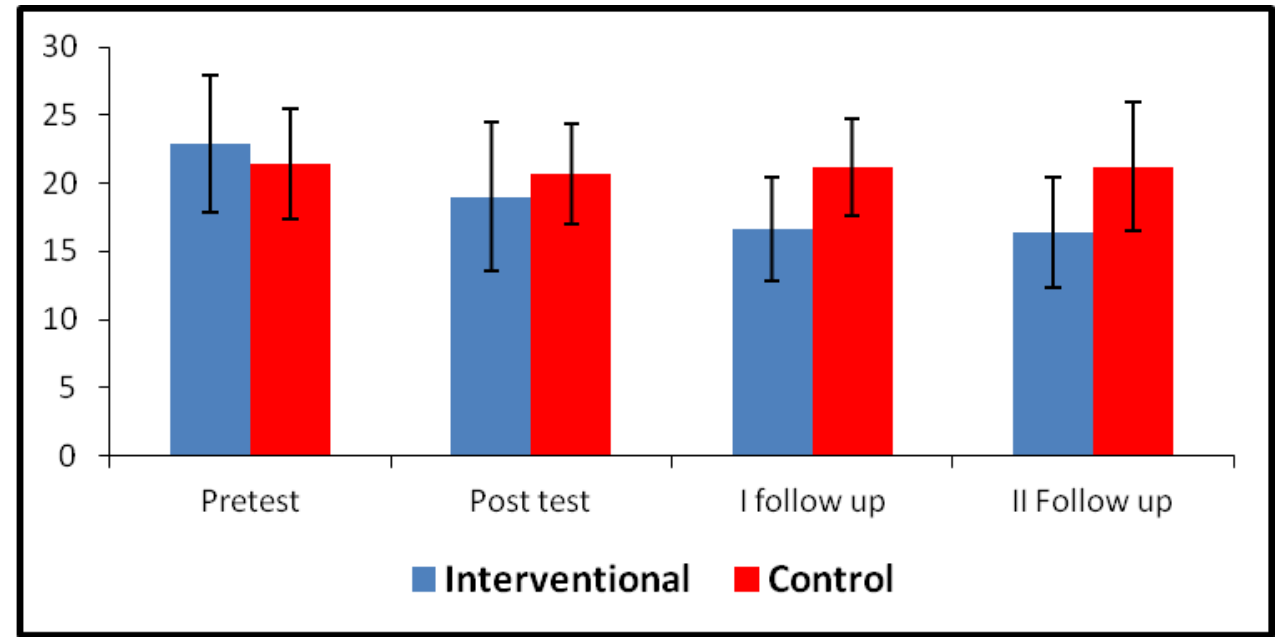

Figure 3. Error plot diagram on depression at various points vs. groups

There is significant difference in the mean of distress, depression, and quality of life between the interventional and control groups. The overall participation in bibliotherapy was $75.3 \%$. The partial eta square value obtained for QoL, psychological distress and depression is 0.2 , 0.60 , and 0.39 , respectively. In conclusion, patients who received bibliotherapy with standard care increased their quality of life and decreased depression and psychological distress than the control group.

\section{DISCUSSION}

Half of the patients were between 46-55 years of age in both the interventional group (52\%) and control group (56\%). Most of the patients were males $(78 \%$ interventional group and $74 \%$ - control group) and married (88\% interventional group and $82 \%$-control group). The monthly income was less than Rs.5000 in $40 \%$ and $52 \%$ of patients in the interventional and control groups, respectively. Fifty percent of them had studied up to secondary education (58\%-interventional group and 54\%-control group). Most of them were suffering from chronic leg and foot ulcer for more than one year (40\% -interventional group and 46\%control group).

Around half of the patients supported themselves economically (48\% - interventional group and 50\% -control group). The spouse was the caregiver for $60 \%$ of the patients in the interventional group, and in control group, the son or daughter was the caregiver for $52 \%$ of the patients. About $28 \%$ of patients in the interventional group were unemployed and $60 \%$ were unemployed in the control group. The Chi- square analysis confirmed that the socio- demographic characteristics were homogenous ( $p>0.05)$.

Most of the patients were struggling with diabetic foot ulcer $(80 \%$ interventional group and $72 \%$ - control group). Injury or trauma $(52 \%$ interventional group and $48 \%$ - control group) was the leading cause for the ulcer. Most of the patients' ulcer was by second episode $(84 \%$ - interventional group and $80 \%$ - control group) and had a single ulcer $(80 \%$ - interventional group and $84 \%$ control group). In interventional group, $78 \%$ of them had undergone incision and drainage, and in the control group, $82 \%$ of them had undergone debridement. Overall, $36 \%$ and $28 \%$ had undergone amputation either in single or two or more toes in both interventional and control groups, respectively.

These socio-demographic and wound related variable distributions were also supported by a prospective study over 3 
years of 60 patients with chronic venous ulcer in Nigeria, which reported that the common age groups were 30-39 and 50-69 years. Also, $93.3 \%$ had unilateral ulcers. Poorly managed traumatic wound was the leading etiological factor for venous leg ulcer (11). A study conducted in a Tertiary Care Hospital India, supported the higher incidence of diabetic foot ulcer than venous ulcer $^{(12)}$.

\section{Impact of Chronic leg and foot ulcer}

The pre - test assessment reported that low quality of life (mean 156.02 with $\mathrm{SD}$ of 5.11), moderate to severe psychological distress (mean 30.12 with SD of 5.11), and moderate depression (mean 22.13 with SD of 4.6). These findings were also supported by a cross - sectional survey on the prevalence of depressive symptoms, diabetes self-management, and Quality of life (Qol) in people with diabetic foot ulcers. All the patients had moderate $(41 \%)$ and low quality of life (59\%). Majority of them reported moderate $(29 \%)$ to severe $(57 \%)$ level of psychological distress .Most of them fell in the borderline $(38 \%)$ and moderate $(56 \%)$ level of depression. Fourteen (23.3\%) reported mild symptoms of depression and 17 (28.3\%) with moderate to severe depressive symptoms ${ }^{(13)}$.

\section{Effectiveness of bibliotherapy on Quality of life}

The quality of life significantly increased after the administration of bibliotherapy in all the five domains The overall quality of life mean increased from 151.35 to 179.49 in post - test, 193.45 in the I follow - up, and 199.89 in the II follow up. In the control group, the overall quality of life mean increased from 160 in the pre test to 163 in the post - test, 167 in the I follow - up, and 177 in the II follow - up. The Bonferroni correction implied that the mean differences of overall quality of life between all the points (pre - test, post - test, I follow - up, and II follow-up) was significant in relation to each other $(\mathrm{p}<0.00)$. These findings were also demonstrated by a cross - sectional study on the relationship between diabetes selfmanagement and quality of life (QoL) among 80 type- 2 diabetic patients with foot ulcers in Thailand. The analysis revealed that, diabetes self-management had significant association with quality of life ( $p$ $<0.05)$. It was advocated that there is need for promoting self-help information to diabetic foot patients to increase good quality of life ${ }^{(14)}$.

\section{Effectiveness of bibliotherapy on Psychological Distress}

In the interventional group, the mean of psychological distress was 29.3, 22.6, 19.59, and 18.7 in the pre the test, post test, I follow-up, and II follow - up, respectively. The mean of psychological distress in the control group was 30.94, $31.62,33.4$, and 33.61 in the pre - test, post - test , I follow-up, and II follow - up, respectively. After the administration of bibliotherapy, the mean of psychological distress gradually decreased to $18.7\left(\mathrm{~F}_{(3,147)}\right.$ $=92, \mathrm{P}<0.05)$ in the Interventional group. The Bonferroni correction implied that the mean differences of psychological distress between all the points (pre -test, post -test, I follow -up, and II follow - up) were significant in relation to each other $(\mathrm{p}<0.00)$. (Except I follow - up with post test and II follow - up -p>0.05). The findings were also supported by a randomized controlled trial conducted about a self-help manual on psychological distress among 54 individuals with moderate depression in Thailand. The findings showed that the post -test distress scores of the intervention group (19.8 and 17) was lower than those in the control group (22.7 and 22.2). Between the post - test and I follow-up, the distress scores continued to decrease steadily in the interventional group, but only decreased slightly in the control group. The findings affirm the application of bibliotherapy to reduce psychological distress ${ }^{(15)}$. 


\section{Effectiveness of bibliotherapy on Depression}

The depression mean was $22.8,19$, 16.59 , and 16.43 in the pre -test, post -test, I follow - up, and II follow - up, respectively in the interventional group. The mean of depression was $21.38,20.72,21.18$, and 21.21 in the pre - test, post -test, I followup, and II follow - up, respectively in the control group. The $\mathrm{F}_{(3,147)}$ value obtained was 32.3. It showed that there was significant decrease in the level of depression after the administration of bibliotherapy in the interventional group whereas same in the control group. The Bonferroni correction implied that the mean difference of depression between the pre test and post -test was significant to each other $(p<0.00)$, except for the I follow-up and II follow-up. Bibliotherapy was found to be effective in reducing depression. Studies conducted at USA and Thailand was supported the effectiveness of bibliotherapy on depression. Bibliotherapy had significantly lower depressive symptoms $\left(\mathrm{t}_{(41)}=3.96, \mathrm{P}<0.001, \mathrm{~d}=0.45\right)$. Bibliotherapy group (14.3 and 13.5) showed less levels of depression than control group (20.2 and 19.4) in post-test and follow up $\left(\mathrm{F}_{(56)}=\right.$ $6.88, \mathrm{p}=0.002)^{(2)}$.

\section{CONCLUSION}

Impact of chronic leg and foot ulcer equally affects the physical and mental health of the person and also increases the risk of psychological distress and depression. Bibliotherapy is in increasing the quality of life. Quality of life was elevated notably in social life, well - being, global Quality of Life, and satisfaction on Quality of Life domains. However, the physical symptoms were not influenced by bibliotherapy. Bibliotherapy is remarkably effective in reducing the psychological distress of patients suffering from chronic leg and foot ulcer. Bibliotherapy is effective in decreasing depression. It gradually decreased after administration of the intervention.

\section{Recommendations}

- Cost effective analysis can be done among chronic leg and foot ulcer patients.

- The self - help book prepared by the researcher can be evaluated on other psychological outcomes such as resilience and anxiety.

The Government can:

- Conduct a funded foot care screening programme.

- Provide special footwear (free of cost) for diabetic patients.

- Initiate special insurance programme for chronic leg wound patients.

The study suggesting that educating care of chronic leg and foot ulcer prevents risk of recurrence and amputation. Mental health issues need to be focused on as early as possible. Psychosocial intervention based on coping, removal of negative cognition, and problem solving to reduce mental health burden in patients with chronic leg wounds. Continuous screening and awareness about the health issues helps in early prevention of depression and other associated risks of chronic leg wounds.

\section{Limitations of the Study}

- The setting was limited to the surgical ward of selected hospitals.

- The data was collected during hospitalization.

- Interventional and control groups were selected from the same setting.

\section{ACKNOWLEDGEMENT}

This acknowledgement submitting heartfelt gratitude to my guide, evaluators and participants of this study of my $\mathrm{PhD}$ thesis. Here I am deeply indepted my acknowledgement to the Nitte (deemed to be) University, Mangalore, India for the opportunity to complete my $\mathrm{PhD}$ study. Special thanks to the staff of K.S.Hegde Medical Academy, Mangalore, India.

\section{Declaration Of The Interest}

Submission of the acknowledgement I express sincere thanks to the secretary and 
Kirupa. P et.al. Effectiveness of bibliotherapy on quality of life, distress and depression among chronic leg and foot ulcer.

director of Indra Gamesman College of Nursing, Tiruchirapalli, India for their constant support and motivation.

\section{Source of Funding: None}

\section{Ethical Approval: Approved}

\section{REFERENCES}

1. LorettaVileikyte. The psycho social impact of diabetes foot damage.Diabetes Voice. Volume 50 November 2005;50:11-13.

2. WallapaSonprugan. Evaluation of a cognitive behavioral bibliotherapy self-help intervention program on the promotion of resilience in individuals with depression. Victoria university,[Melbourne]; 2010. Available from: https://www. researchgate.net/ 43440460.

3. Edwards H. Identification of symptom clusters in patients with chronic venous leg ulcers. Journal of Pain Symptom Management. 2014 May;47(5):867-75. Available from: 10.1016/j.jpainsymman.2013.06.003.E-pub 2013Aug 30.

4. Müller S, Rohde P, Gau JM and Stice E. Moderators of the effects of indicated group bibliotherapy and cognitive behavioral depression prevention programs on adolescent's depressive symptoms and depressive disorder onset. Behaviour Research and Therapy. 2015 Dec; 75:1-10.

5. Kirupa.P, B.PreethamRai, Shrinivasa Bhat.U. Systematic review on Impact of chronic leg wounds: a step to develop theory. International Journal Nursing Education and Research.2014JulySeptember; 2(3): 258-63.

6. Neeru B, Gagandeep K, Pal AJ, Bajwa SS, Harbandna S, Rajesh K. Psychosocial, psychiatric, and clinical implications of diabetic foot ulceration: A prospective analysis. Journal Social Health and Diabetes.2015; ; 3 (89-94.

7. Berger T, Hämmerli K, Gubser $\mathrm{N}$, AnderssonG and Caspar F.Internetbased treatment of depression: a randomized controlled trial comparing guided with unguided self-help. Cognitive Behavior Therapy. 2011;40(4):251-66.

8. KirupaP, PreethamRai B., and Shrinivasa BhatU. A Pilot Trial: Effectiveness of bibliotherapy on quality of life, psychological distress and depression among patient with chronic leg and foot ulcer in Mangalore. Nitte university Journal of Health sciences .March 2015; Vol 5,No 1 :9-13.

9. Kothari.Research methodology. $2^{\text {nd }}$ revised edition. New Age International Publishers; 2004.

10. Discovering statistics using IBM SPSS Statistics:And sex and drugs and rock 'n' roll $.4^{\text {th }}$ edition.Sagepublication.www.discovery of statistics .com35.

11. Rahman GA, Adigun IA, and Fadeyi A. Epidemiology, etiology, and treatment of chronic leg ulcer: experience with sixty patients. Annals of African Medicine. 2010 Jan-Mar ; 9(1) : 1-4.

12. Gupta N,Gupta .S.K,Shukla.V.K, and Singh SP. An Indian community-based epidemiological study of wounds. Journal of Wound Care. 2004 September; 13(8):323-5.

13. Pearson S, Nash T, Ireland V. Depression symptoms in people with diabetes attending outpatient podiatry clinics for the treatment of foot ulcers. Journal of Foot and Ankle Research. 2014 November 25;7(1):47

14. Navicharen .R. Diabetes self-management, fasting blood sugar and quality of life among type 2 diabetic patients with foot ulcers. Journal of Medical Association of Thai. 2012 May; 95(5):746.

15. SongprakunWand McCann TV. Evaluation of a bibliotherapy manual for reducing psychological distress in people with depression: a randomized controlled trial Journal of Advanced Nursing 2012 Dec; 68(12):2674-84 . doi: 10.1111/j.13652648.2012.05966.x.

How to cite this article: Kirupa. P, Shrinivasa Bhat. Effectiveness of bibliotherapy on quality of life, distress and depression among chronic leg and foot ulcer. Int J Health Sci Res. 2021; 11(4): 93-101. DOI: https://doi.org/10.52403/ ijhsr.20210412 